\title{
EDIFİCATE
}

I Congreso de Escuelas de Edificación y Arquitectura Técnica de España

València, 4 y 5 de noviembre de 2021

Escuela Técnica Superior de Ingeniería de Edificación

Universitat Politècnica de València

Doi: https://doi.org/10.4995/EDIFICATE2021.2021.13592

\section{La metodología BIM como elemento vehicular en la docencia de Grado en Ingeniería de Edificación}

\section{BIM as a teaching tool in Building Engineering Degree}

\author{
David Valverde Cantero ${ }^{a}$ \\ aEscuela Politécnica de Cuenca, Universidad de Castilla-La Mancha, david.valverde@uclm.es
}

\begin{abstract}
Although, fortunately, we agree on the need to implement BIM methodology in university education effective strategies are still necessary to solve problems derived from such a big change. The strategy adopted by Escuela Politécnica de Cuenca was proposed with a transversal integration of BIM methodology and always based on the generosity of the agents -teachers and students-, in the absence of hierarchies and that seeks the motivation of the students.

The different experiences developed in recent years, an attempt has been made to promote collaborative work with challenges and joint workshops and overcoming the traditional teacher-student roles.
\end{abstract}

Keywords: BIM, teaching methodology, Building Engineering Degree, implementation, motivation, collaborative work.

\section{Resumen}

Aunque la necesidad de implementación de la metodología BIM en el ámbito de enseñanza universitaria ya parece, afortunadamente, un debate superado siguen siendo necesarias estrategias eficaces que solventen los problemas derivados de un cambio tan profundo. La estrategia de la Escuela Politécnica de Cuenca se planteó con una integración transversal en varios frentes, pero siempre basada en la generosidad de los intervinientes -profesores $y$ alumnos-, en la ausencia de jerarquías y en la motivación del alumnado.

En las distintas experiencias llevadas a cabo durante los últimos años se ha conseguido fomentar el trabajo colaborativo con retos y talleres conjuntos $y$ superando los roles tradicionales profesor-alumno.

Palabras clave: BIM, metodología docente, Ingeniería de Edificación, implementación, motivación, trabajo colaborativo. 


\section{Introducción}

La implementación de la metodología BIM ha sido interpretada en la Escuela Politécnica de Cuenca -EPCu- (Escuela Politécnica de Cuenca, 2021) como una oportunidad para organizar las acciones que en esta línea se venían realizando desde el curso 2017-2018 y proponer otras nuevas dentro del entorno del trabajo colaborativo, en un marco único para analizar y evaluar diferentes acciones para promover y profundizar en la filosofía BIM en el seno del Grado en Ingeniería de Edificación -GIE- (Oliver, 2015).

En este contexto destaca la iniciativa "aula BIM EPCu" (Fig. 1) como apoyo/afianzamiento a la implementación docente de BIM, pero desde un enfoque menos rígido que el propio de las asignaturas del plan de estudios (Valverde, 2019).

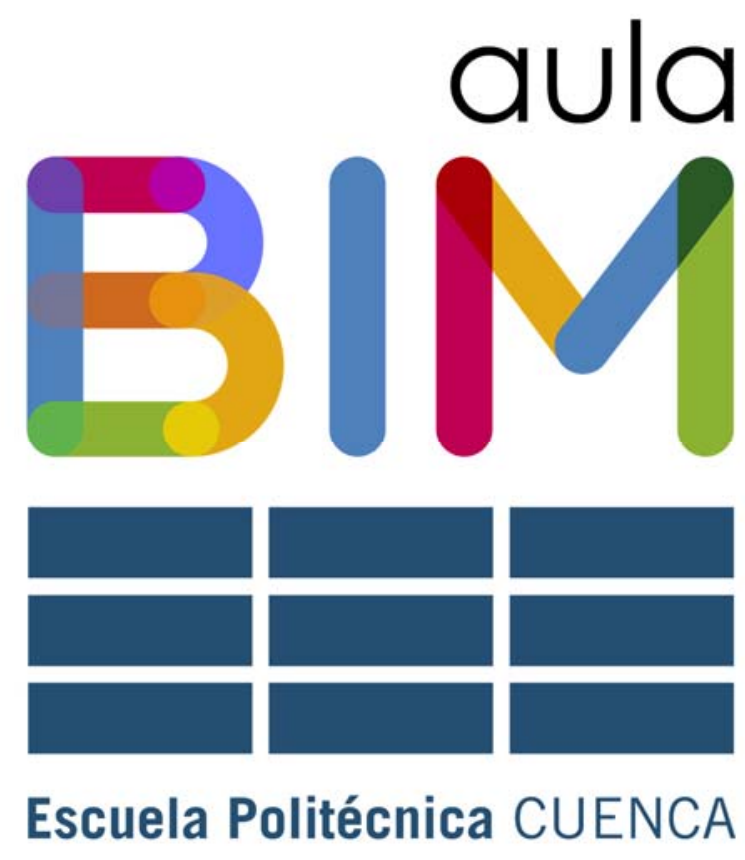

Fuente: Elaboración propia

Fig. 1 Logotipo aula BIM EPCu

Cabe destacar también que muchas de las acciones llevadas a cabo se recopilaron en un Proyecto de Innovación docente (Alfaro et al, 2018) que sirvió de base para plantear una serie de estrategias-recomendaciones prácticas centradas en adquirir destrezas "blandas" que aportan ayuda a ese otro $80 \%$ de metodología BIM que no está estrictamente vinculado con un manejo de software BIM. 


\section{Objetivos}

\subsection{Contenido y metodología docente clásica}

No se pretende cambiar los contenidos, pero sí la metodología. El principal objetivo es el de diseñar y poner en práctica un conjunto de acciones que permitan dotar de carácter integrador las técnicas de trabajo colaborativo BIM en el GIE. Se pretende con ello que las experiencias desarrolladas sirvan como ejercicios integradores de calado transversal dentro de la titulación, además de permitir la elaboración y desarrollo de prácticas docentes innovadoras que supongan la mejora y renovación del proceso enseñanza-aprendizaje de nuestros estudiantes.

\subsection{Metodología docente complementaria}

Admitiendo que efectuar un cambio completo y simultáneo no es viable apostamos por una vía paralela/complementaria que propone una metodología de trabajo colaborativa (AgullódeRueda, 2018) que esté coordinada entre las distintas asignaturas y/o materias interesadas.

Este entorno debe servir para recoger/filtrar/utilizar los avances/incidencias que, propuestos tanto por el profesorado como por los alumnos, estén relacionados con el desarrollo de cada una de las asignaturas y cristaliza en talleres/cursos específicos o en la participación de concursos BIM.

\section{Desarrollo de la innovación}

Centrándonos en las actividades desarrolladas bajo el concepto de aula BIM EPCu durante los últimos cuatro cursos podemos decir que las mismas han sido, preferentemente, presenciales. Aunque también han existido solicitudes/ensayos para la retransmisión de las mismas vía streaming sólo durante el último curso y medio, y debido a la crisis sanitaria por COVID-19, se ha recurrido a esta modalidad ya que entendemos que carece de las posibilidades de las sesiones presenciales.

Además, la demanda de actividades ha sido fundamentalmente bajo el formato de ASESORÍAS puntuales atendidas por el becario del aula BIM sobre temas muy concretos y a grupos reducidos.

Por otro lado, los TALLERES han sido propuestos/ programados mayoritariamente por los responsables de la iniciativa intentando no coincidir con otras actividades -ciclo de conferencias, periodos de exámenes, reuniones de carácter general...- e intentando garantizar unas asistencias mínimas. Para estos TALLERES se ha contado tanto con el personal docente de la EPCu o del resto de la UCLM, como con antiguos alumnos y con otros profesionales de reconocido prestigio que han accedido a compartir sus conocimientos en un entorno abierto a la participación de los asistentes. 


\section{Resultados}

De manera paralela a la realización de las actividades del aula BIM EPCu se ha llevado un minucioso registro y monitorización, mediante encuestas anónimas, donde los asistentes pudieran valorar la iniciativa. Los datos recopilados se cruzaron con los registros de asistencias para arrojar los datos que se muestran a continuación.

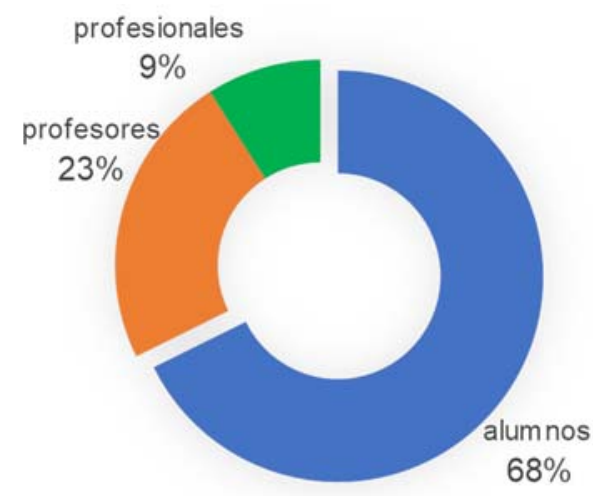

Fuente: Elaboración propia

Fig. 2 Perfil de asistente al aula BIM EPCu 2017-2021

En la primera gráfica (Fig. 2) se puede observar el perfil de acceso de los asistentes a las distintas sesiones, con un total de 869 asistentes, incluidos recurrentes, en los cuatro cursos de vida de aula BIM EPCu.

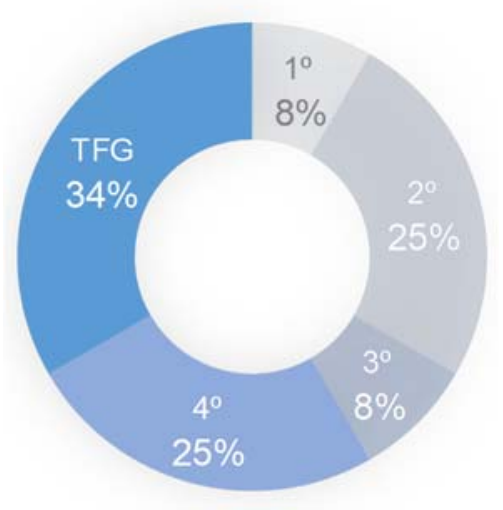

Fuente: Elaboración propia

Fig. 3 Perfil de alumnos asistentes al aula BIM EPCu 2017-2021

Si desglosamos los datos de asistencia general para los 591 alumnos participantes (Fig. 3) vemos que los estudiantes que hacen uso del aula se encuentran distribuidos de manera homogénea a lo largo de todos los cursos -aclarando que la baja asistencia de alumnos de $1^{\circ}$ se debe a la baja matriculación en el mismo durante los últimos años-. 
En cuanto a los profesores asistentes cabe destacar que fundamentalmente han sido respaldados por el aula BIM mediante el formato de ASESORÍAS. En ellas han podido desdoblar sus clases y hacer que los alumnos se inicien en herramientas BIM con las que poner en práctica los conocimientos adquiridos en clase. Los TALLERES también han servido para que profesores de otras áreas hayan entrado en contacto con los diferentes enfoques que propone la metodología BIM.

Por último, bajo el epígrafe de profesionales la EPCu también ha dado cabida a perfiles con mayor experiencia laboral/aplicada -antiguos alumnos en su mayoría- que ha aprovechado la iniciativa para compartir su experiencia con el resto de los asistentes y/o comenzar su reciclaje profesional bajo esta nueva metodología BIM.

\section{Conclusiones}

Aunque se trata de una iniciativa que ha ido puliéndose año a año, cabe resaltar la predisposición/buena acogida por parte del alumnado -con una nota media de 4,6 sobre 5y también, al menos en parte, entre el profesorado. Iniciativas como esta, esperamos, deben ejercer de dinamizadoras en el proceso de adopción de metodología BIM en el plan de estudios de GIE.

También entendemos que el progreso de contenidos/adhesiones por parte de alumnos/ profesores no puede ni debe ser impositivo, sino que más bien se trata de una "imparable expansión en mancha de aceite". La evolución de los índices de asistencia así lo demuestra y la ampliación de contenidos, diversidad de ponentes y participación de antiguos alumnos y profesionales, puede hacer de esta iniciativa una fuente de difusión, aprendizaje y testado de conocimientos, necesaria y complementaria a la formación reglada.

Nuestra iniciativa, supone una voluntad de implementación de la metodología BIM que, afortunadamente, es ajena a las voluntades de los responsables de las asignaturas. Estamos extendiendo una metodología aplicable en cualquiera de las asignaturas, lo deseable sería un entorno en el que todos estuvieran a favor y la colaboración y la participación fueran algo natural. Pero, entendiendo que esto puede que no siempre sea así, hemos diseñado un procedimiento que, sin estorbar ni imponer, permita la implementación por convencimiento y experimentación.

Queda claro también que es imposible afrontar problemas complejos en un entorno colaborativo si no se ha participado previamente en talleres y simulacros durante el proceso de aprendizaje. El profesor y el alumno deben ser conscientes cuanto antes de esa mentalidad y de adecuación de la metodología BIM a la misma. 


\section{Referencias}

ESCUELA POLITÉCNICA DE CUENCA. Grado en Ingeniería de Edificación. $<$ https://www.uclm.es/cuenca/epc/gradoedificacion > [Consulta: 30 de junio de 2021]

OLIVER FAUBEL, I. (2015). Integración de la metodología BIM en la programación curricular de los estudios de Grado en Arquitectura Técnica/Ingeniería de Edificación. Diseño de una propuesta. Tesis Doctoral. Valencia: Universitat Politècnica de València, < https://riunet.upv.es/handle/10251/61294> [Consulta: 30 de junio de 2021]

VALVERDE CANTERO, D, PÉREZ GONZÁLEZ, PE y ALFARO GONZÁleZ, J. (2019). "La implementación BIM mediante el aula BIM en la Escuela Politécnica de Cuenca" En: XIV Congreso Internacional de Expresión Gráfica Aplicada a la Edificación APEGA 2019. Sevilla: Editorial Círculo rojo. $48-50$.

ALFARO GONZÁLEZ, J, VALVERDE CANTERO, D, CAÑIZARES MONTÓN, JM, MARTíNEZ CARPINTERO, JA y PÉREZ GONZÁLEZ, PE. (2018). "EI BIM-LaB como aglutinador de la experiencia de implantación BIM académica y científica para profesores, alumnos y exalumnos de la Escuela Politécnica de Cuenca. UCLM" En: The international BIM conference, EUBIM 2018. Valencia: Editorial Universitat Politècnica de València. 57-66.

AGULLÓ-DERUEDA, J, JURADO-EGEA, J, INGLÉS-GOSÁLBEZ, B. (2018). "Trabajos colaborativos BIM en enseñanzas de grado" En: The international BIM conference, EUBIM 2018. Valencia: Editorial Universitat Politècnica de València. 35-46. 